\title{
Polystyrene Latex Particles Bearing Primary Amine Groups via Soap-free Emulsion Polymerization
}

Pascal Marmey ${ }^{\mathrm{a}}$, Noureddine Lebaz ${ }^{\mathrm{b}}$, Mohamed Eissa ${ }^{\mathrm{b}, \mathrm{c}}$, Thierry Delaird, Abdelhamid Elaissari ${ }^{\mathrm{b}, *}$

${ }^{a}$ Unité mixte CNRS-BioMérieux, Ecole Normale Supérieure de Lyon, 46, allée d'Italie 69364 Lyon, France

${ }^{b}$ Univ Lyon, University Claude Bernard Lyon 1, CNRS, LAGEPP UMR 5007, 43 boulevard du 11 novembre 1918, F-69100 Villeurbanne, France

${ }^{c}$ Polymers and Pigments Department, National Research Centre, 33 El Bohouth St. (Former El Tahrir St.), Dokki, Giza 12622, Egypt

${ }^{d}$ Univ Lyon, University Claude Bernard Lyon 1, CNRS, IMP, UMR 5223, 15 Boulevard Latarjet, F-69622, Villeurbanne, France

Corresponding Author:*E-mail: abdelhamid.elaissari@univ-lyon1.fr

\begin{abstract}
Polystyrene latexes were prepared in the presence of an amino-containing functional comonomer, i.e. N-(3-aminopropyl) methacrylamide hydrochloride (APMH), via soap-free batch emulsion polymerization initiated by the cationic initiator 2,2'-azobis (2-amidino propane) di-hydrochloride (V50). These latexes were characterized through studying the influence of ionic comonomers on the polymerization kinetics, particle size, surface charge density and colloidal properties. The synthesized latexes were monodisperse with a final size between 100 and $600 \mathrm{~nm}$ depending on the APMH concentration. The initial polymerization rate and the particles number increased in accordance with the Smith-Ewart theory for soapfree styrene emulsion polymerization with a hydrophilic functional comonomer. The final functionalization rate of the particles has been particularly studied with the intention of fitting the prepared latexes to be utilized in the immobilization of biological molecules for biological sample preparation and diagnostic applications.
\end{abstract}

This article has been accepted for publication and undergone full peer review but has not been through the copyediting, typesetting, pagination and proofreading process which may lead to differences between this version and the Version of Record. Please cite this article as doi: $10.1002 /$ pi.6060 
Keywords: Polystyrene latexes, Emulsion polymerization, Functionalized particles, Amine groups

\section{INTRODUCTION}

Polymer dispersions with controlled physical, physico-chemical and colloidal properties are the subject of intensive research since many decades because of their wide range of applications. ${ }^{1}$ Among these polymer dispersions, functional polymer particles bearing surface reactive groups are employed in many fields including chromatography (as column-filling materials), ${ }^{2-4}$ biosensors, ${ }^{5,6}$ contrast agents for medical imaging, ${ }^{7-9}$ medical diagnostics, ${ }^{9,10}$ and drug delivery. ${ }^{11}$ The functional surface reactive group may be a carboxyl group, ${ }^{12-14} \mathrm{a}$ hydroxyl group, ${ }^{15}$ an ester group, ${ }^{16,17}$ an amine group, ${ }^{18-22}$ a protein, ${ }^{23,24}$ or any other specific group depending on the intended application.

Polymerization methods such as emulsion, miniemulsion, microemulsion, and dispersion polymerizations are generally adopted to produce polymer dispersions with target specifications which are mainly particle size, particle size distribution, surface charge density, and presence of surface reactive groups. ${ }^{25,26}$ Polystyrene latex particles are highly investigated because of their low cost and relative stability. ${ }^{27}$ Because of their hydrophobicity and their low adhesive properties, polystyrene microspheres have been synthesized with a surface functional group in order to strongly bind or chemically react with a specific molecule. ${ }^{28}$ This study focused on amino-functionalized polystyrene latexes.

Amino groups are used as functional groups for the covalent immobilization of biologically active macromolecules. ${ }^{29}$ They confer a cationic character to the hosted particles. The synthesis of amino-functionalized latexes is possible via different strategies. The first method 
is the chemical modification of other surface functional groups such as carboxyl, hydroxyl, amide or chloromethyl into amino groups. ${ }^{19,27,30-32}$ This method suffers from the instability of the colloidal system as reported by Forcada and Hidalgo-Álvarez (2005). ${ }^{28}$ A direct method is possible by emulsion copolymerization of amine-functional monomers with styrene. ${ }^{33-35}$ Following this method, Ganachaud et al. (1997) have investigated the kinetics of batch emulsifier-free emulsion copolymerization of styrene in the presence of two amino-containing monomers, aminoethyl methacrylate hydrochloride (AEMH) and vinyl benzyl amine hydrochloride (VBAH). ${ }^{33}$ They discussed the polymerization mechanism in presence of AEMH and VBAH. Sauzedde et al. (1997) reported the colloidal and surface properties of these copolymer latex particles. ${ }^{34}$ Polystyrene particles bearing amino groups may be prepared also by a two-step process consisting of copolymerization of the amino-functional monomer in the presence of polystyrene seed particles. ${ }^{20,21,36-38}$ The first step consists of synthesizing anionic polystyrene monodisperse latex particles which are used as seed. In the second step, styrene and amino-functional monomers are copolymerized in presence of the previously formed latex particles. The cationic amino-functional monomers used are 4vinylbenzylamine (VBA) and its trifluoroacetamide derivatives (VBAF), AEMH, and VBAH. It is worth mentioning that all the above discussed studies are related to emulsion polymerization. Amino-functionalized nanoparticles were also prepared by miniemulsion polymerization as well. ${ }^{39-41}$

In this study, polystyrene latex particles bearing primary amine groups were synthesized via soap-free emulsion polymerization. The effect of functional ionic monomer concentration on polymerization rate and on particles size and number were investigated. In addition, the 
amounts of accessible surface amino groups were determined experimentally and discussed. Colloidal stability of the different synthesized latexes was also examined in terms of zeta potential measurements as a function of $\mathrm{pH}$ of the dispersion medium.

\section{EXPERIMENTAL SECTION}

\subsection{Materials}

Styrene monomer (99 \% pure) was from Janssen and distillated under vacuum conditions. The $\mathrm{N}$-(3-Aminopropyl) methacrylamide hydrochloride (APMH) was from Kodak and used as received (Figure 1). 2,2'-azobis amidinopropane dihydrochloride (V50) was from Wako and purified by recrystallization. 4-dimethylamino-pyridine (DMAP) from Aldrich, 3-(2pyridyldithio)-propionic acid N-hydroxysuccinimide ester (SPDP) and DL-dithiothreitol (DDT) from Sigma were used as received. Water was purified by a Millipore Q-MT system and it was boiled under nitrogen stream during 2 hours before use in the latex preparation.

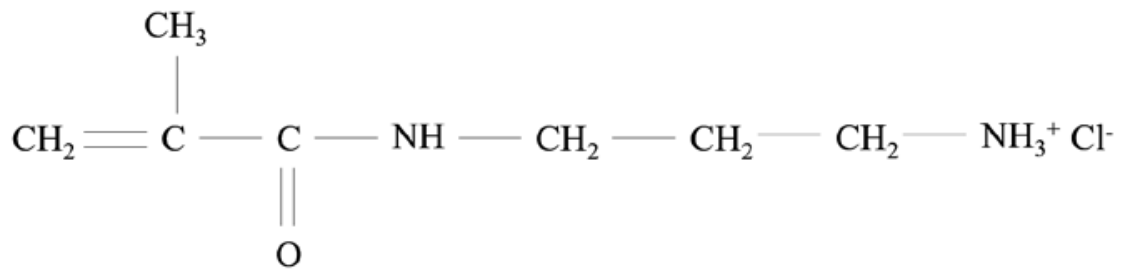

Figure 1. Chemical structure of APMH: N-(3-Aminopropyl) methacrylamide hydrochloride.

\subsection{Preparation of latexes}

Polymerization experiments were performed using batch polymerization process. All ingredients used are shown in Table 1 and the polymerization was carried out in a four-necks glass reactor equipped with a glass paddle-type agitation, condenser and nitrogen inlet. The 
polymerization temperature was controlled at $70{ }^{\circ} \mathrm{C}$. Agitation was fixed at $300 \mathrm{rpm}$. The obtained latexes were cleaned by repetitive centrifugation using deionized water in order to remove free electrolytes and water-soluble polymers before any colloidal characterization.

Table 1. Reagents used for the latexes preparation by surfactant free radical emulsion polymerization

\begin{tabular}{|l|c|}
\hline Reagents & Quantities (g) \\
\hline Water & 200 \\
Styrene monomer & 10 \\
V50 initiator & 0.55 \\
APMH & from 0 to 1.37 \\
\hline
\end{tabular}

\subsection{Characterization of Latexes}

\subsubsection{Water-Soluble Polymer (WSP)}

The water-soluble polymer (WSP) amount was quantified thermogravimetrically after separation from latex particles via a centrifugation process.

\subsubsection{Polymerization kinetics}

The polymerization was followed by collecting samples at various reaction times in which hydroquinone traces were added to stop the reaction. Then, the collected samples were stored at $4{ }^{\circ} \mathrm{C}$ before analysis. The polymerization conversion was determined by thermogravimetric analysis.

\subsubsection{Particle size and distribution}

Particle size was measured both by Quasi Elastic Light Scattering (QELS) and by Transmission Electron Microscopy (TEM). The QELS was investigated using Zeta Sizer 3000 
HS and NanoZS from Malvern Instruments. The intensity autocorrelation function induced by particles scattering light as they undergo Brownian motion is fitted using CONTIN algorithm to get access to the particle size distribution. Because of the low polydispersity index (PDI) in our case, only the mean diameters are reported. The measurements were performed at least four times and the reported values are the average ones.

TEM analysis was performed using a Hitachi S 800 (CMEABG at Claude Bernard University, Lyon I, France). This method gives information on particle size and size distribution as well as morphology of the latex particles under dried state. Samples were prepared by placing a drop of the dispersions directly onto a grid and then were left to dry at room temperature before TEM examination.

\subsubsection{Electrophoretic mobility}

The electrophoretic mobility of latexes was measured using the Zeta Sizer III and NanoZS, from Malvern Instruments. The experiments were carried out using a very diluted latex solution in $10^{-3} \mathrm{M} \mathrm{NaCl}$. The electrophoretic mobilities were determined as a function of $\mathrm{pH}$ at $20^{\circ} \mathrm{C}$. Each value was obtained by taking the average of at least three measurements.

\subsubsection{Surface charge density}

The surface charge density of the prepared latexes was determined using SPDP method. ${ }^{18}$ This method is based on the chemical reaction between the amine groups present at the latex particles surface and 3-(2 pyridyldithio) propionic acid compound. The principle of the chemical titration method is depicted in the reaction scheme in Figure 2. 


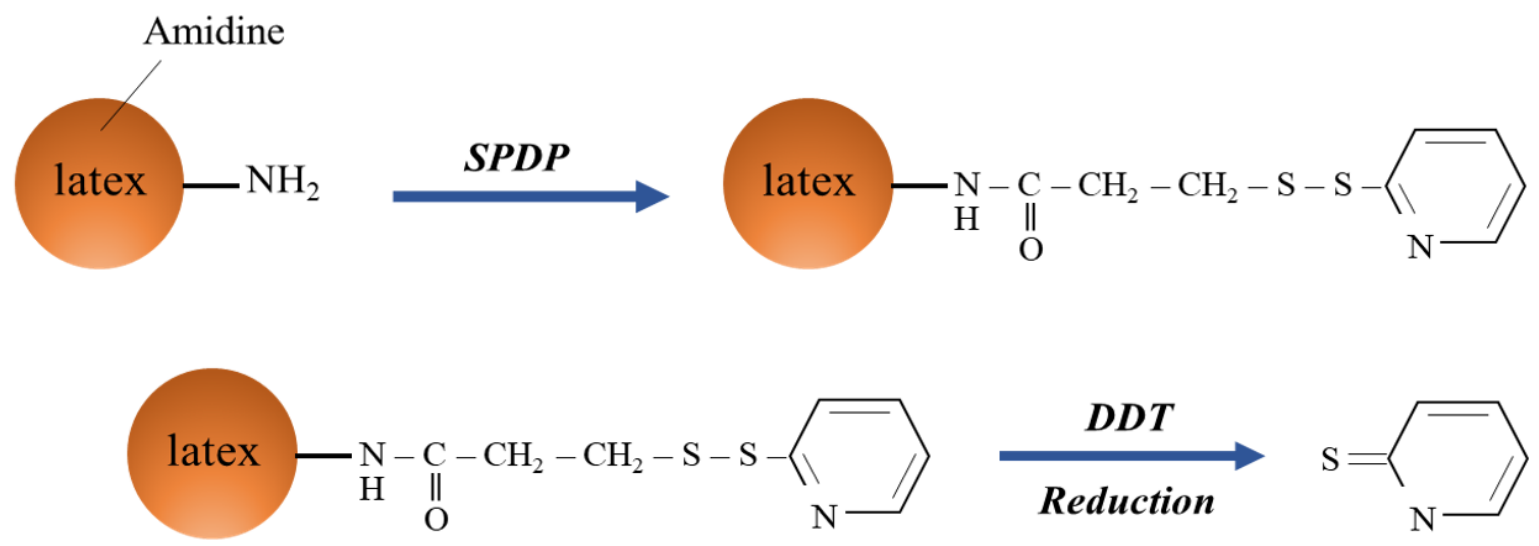

Figure 2. Schematic illustration of SPDP titration method based on chemical reaction of amine groups used for the determination of surface charge density of the synthesized latex particles.

\section{RESULTS AND DISCUSSION}

\subsection{Preparation of latex particles}

\subsubsection{Kinetic study}

The influence of the cationic functional monomer (APMH) concentration on the polymerization kinetics of styrene has been investigated. The concentration of APMH was varied from 0 to $1.37 \mathrm{~g}$ (i.e. from 0 to $3.85 \times 10^{-2} \mathrm{~mol} \mathrm{~L}^{-1}$ ). The amount of styrene used in all the polymerization experiments was fixed at $2 \%$ molar referred to styrene plus APMH monomer. The conversion-time curves of styrene and APMH were followed as represented in Figure 3. 


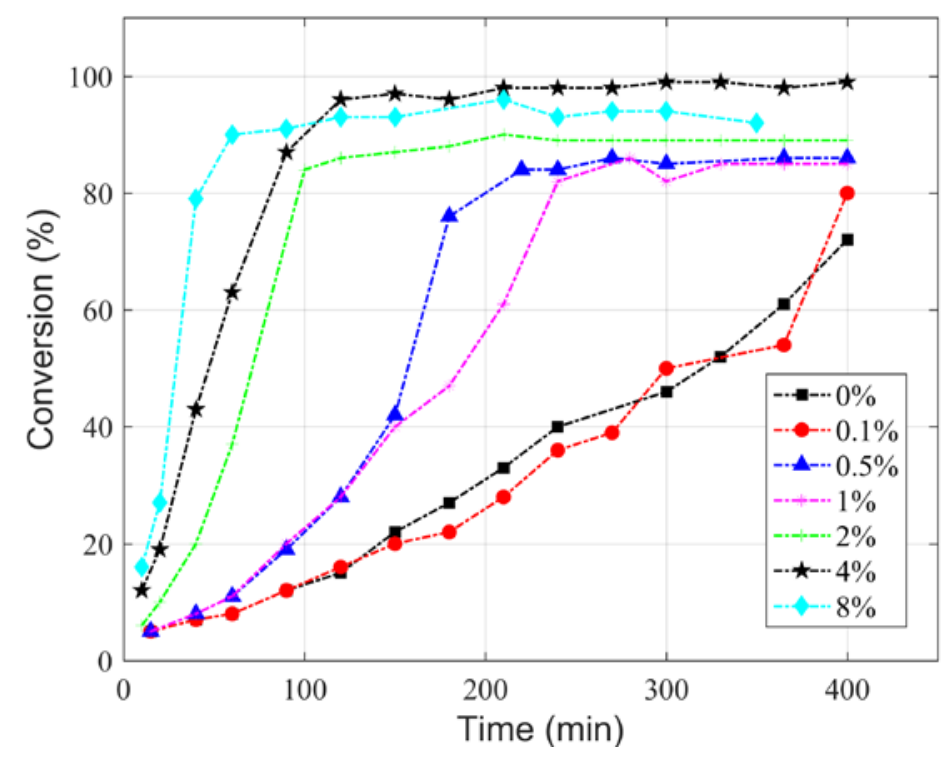

Figure 3. Percentage conversion versus time as a function of APMH concentration (\% molar) referred to styrene in the polymerization reaction mixture.

It seems that the higher the concentration of APMH, the steeper the initial polymerization rate, indicating that the cationic monomer would cause the formation of a larger number of polymerization loci. This is confirmed through the examination of both the final particles size and particle size distribution variation as a function of polymerization time. In fact, as shown in Figure 4, all latexes appear quite monodisperse, which suggests an extremely short nucleation step and confirmed through the examination of Figure 5 which shows the formation of monodispersed particles in less than 50 minutes (for low APMH monomer concentration). 

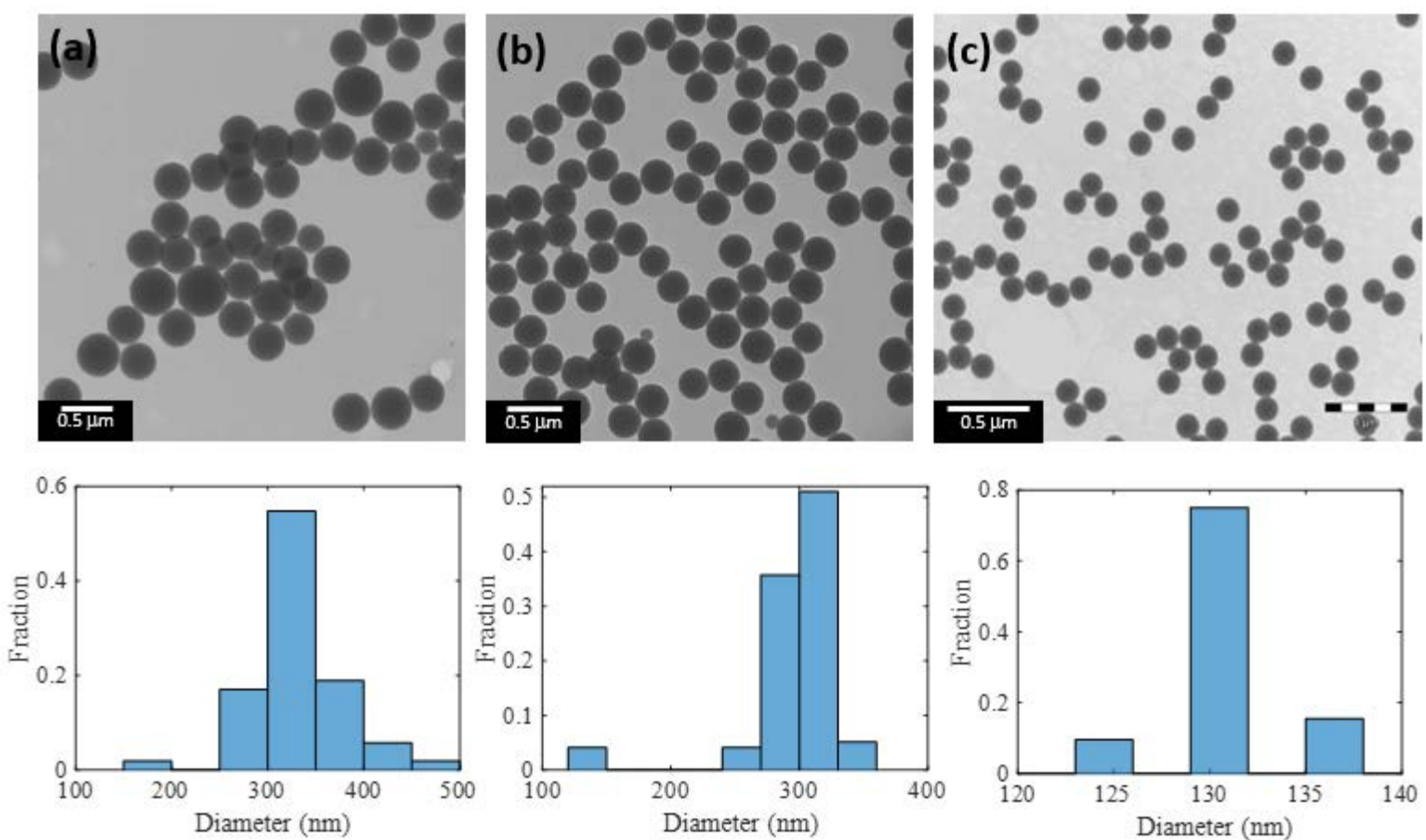

Figure 4. TEM images and their corresponding histograms of the as-prepared polystyrene particles using (a) PAMH free, (b) $0.5 \%$ molar APMH and (c) $4 \%$ APMH monomer (scale bar is $500 \mathrm{~nm}$ ).

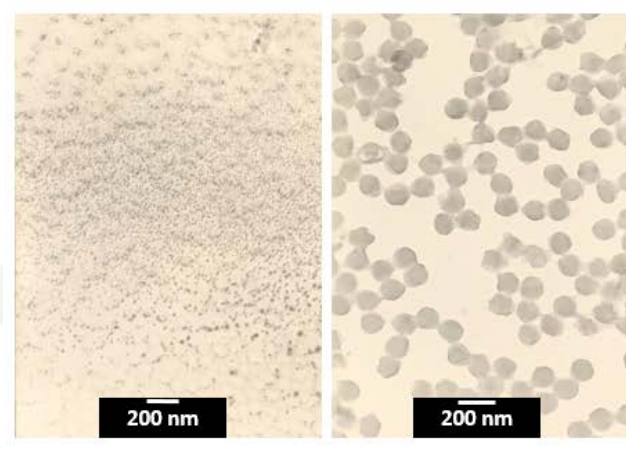

$5 \min$

$30 \mathrm{~min}$

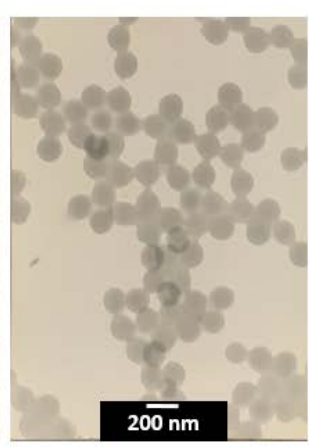

$45 \mathrm{~min}$

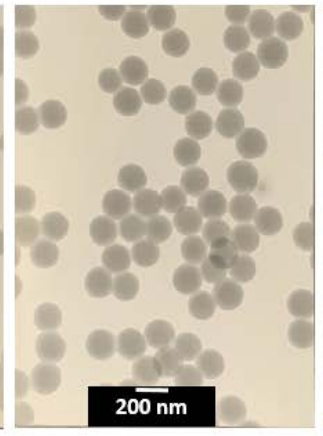

$75 \mathrm{~min}$

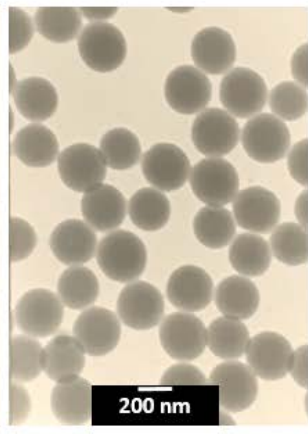

$300 \mathrm{~min}$

Figure 5. TEM images of latex particles (for $0.5 \%$ APMH amount) at various polymerization times (all micrographs have the same magnification; scale bar is $200 \mathrm{~nm}$ ).

\subsubsection{Effect of functional monomer concentration on polymerization rate}


The influence of APMH monomer concentration on the initial polymerization rate $\left(R_{p}\right)$ is derived from the slope of conversion versus time curves as reported in Figure 6. In fact, the initial polymerization rate deduced from the conversion - time curves can be related to the propagation constant and particles number according to Smith-Ewart theory ${ }^{42,43}$ illustrated by the following equation (1):

$$
R_{p}=k_{p} \times[M] \times \frac{N_{p}}{N_{a}} \times \bar{n}
$$

where $k_{p}$ is the propagation rate constant and for the propagation of styrene in the particles, this constant rate is $k_{p}=4.8 \times 10^{-2} \mathrm{~L} \mathrm{~mol}^{-1} \mathrm{~s}^{-1}$. [M] is the monomer concentration in the particles $\left([M]=5.8 \mathrm{~mol} \mathrm{~L}^{-1}\right), N_{a}$ is the Avogadro's number $\left(N_{a}=6.02 \times 10^{23} \mathrm{~mol}^{-1}\right), \bar{n}$ is the average number of free radicals per particle ( $\bar{n}=0.5$ in the case of zero-one conditions), $N_{p}$ is the particle number per unit volume of the dispersion.

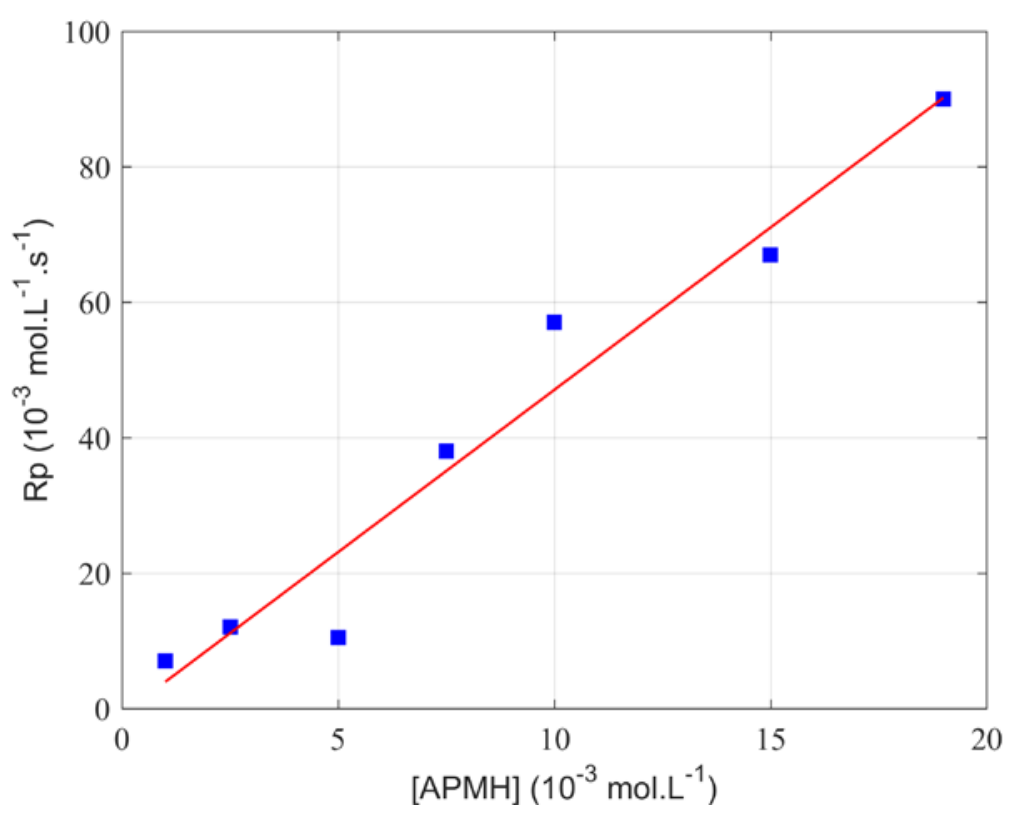

Figure 6. Polymerization rate as a function of the cationic monomer (APMH) concentration. 
The result obtained is given in Figure 6 in which $R_{p}$ displays a relative linear variation versus functional monomer concentration with a positive slope. Such behavior reveals that the increase in the functional monomer concentration in the polymerization recipe increases the initial polymerization rate as generally observed in the case of emulsion polymerization. ${ }^{33}$ In fact, the addition of ionic monomer in the emulsion polymerization influences drastically the polymerization mechanism by affecting principally the nucleation step, i.e. particles number and the average radicals' number in the obtained total particles.

\subsubsection{Effect of functional monomer concentration on particle size and particles number}

As largely reported in the case of soap-free emulsion polymerization of styrene, the final particle size decreases with the addition of an ionic monomer in the polymerization recipe. ${ }^{33,34}$ The particle size evolution depends on several parameters such as conversion, particles number and polymerization rate as illustrated in Figure 7 for $0.5 \%$ functional APMH monomer. 


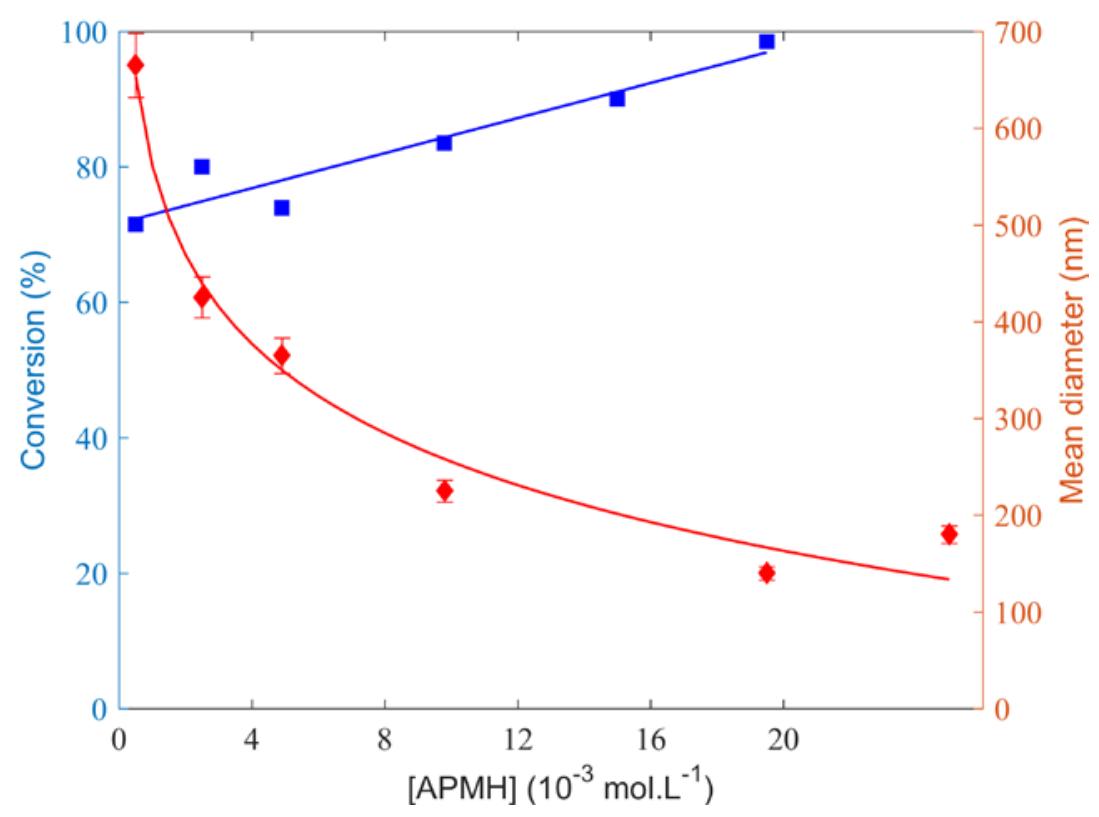

Figure 7. Effect of the ionic monomer concentration on both final mean particle size and conversion.

As shown in Figure 7, the final conversion slightly increases with increasing the ionic monomer concentration as expected. In fact, the ionic monomer promotes not only the polymerization rate but also the total conversion (polymer particles and water-soluble polymer). Whereas, the final particle size decreases when the APMH amount increases. This behavior is principally attributed to the influence of the ionic monomer on particles number which is directly related to the colloidal stability of immature preliminary particles formed at the early stage of the polymerization process. The particles number can be determined from the conversion (after subtracting the amount of water-soluble polymer) and particle size using the following equation (2):

$$
N_{p}=\frac{6 \times T S}{\rho \pi \overline{D_{n}}}
$$


where TS is the solid content of latex particle, $\rho=1.045 \mathrm{~g} \mathrm{~cm}^{-3}$ is the density of polystyrene and $\overline{D_{n}}$ is the number-based average particle size (mean diameter). The above-mentioned particles number can be related to the initiator and ionic monomer concentrations as given by the following empirical equation:

$$
\mathrm{N}_{\mathrm{p}}=\mathrm{k}^{\prime} \times[\mathrm{I}]^{\alpha} \times[\mathrm{A}]^{\beta}
$$

where $\mathrm{k}^{\prime}$ is an arbitrary constant, [I] and [A] are the initiator and the ionic monomer concentrations respectively and $\alpha$ and $\beta$ are the relative coefficients for the initiator and ionic monomer respectively. Since the initiator concentration is constant, the effect of the ionic monomer concentration was evidenced by plotting $\mathrm{N}_{\mathrm{p}}$ versus $[\mathrm{A}]$ in log-log scale as illustrated in Figure 8.

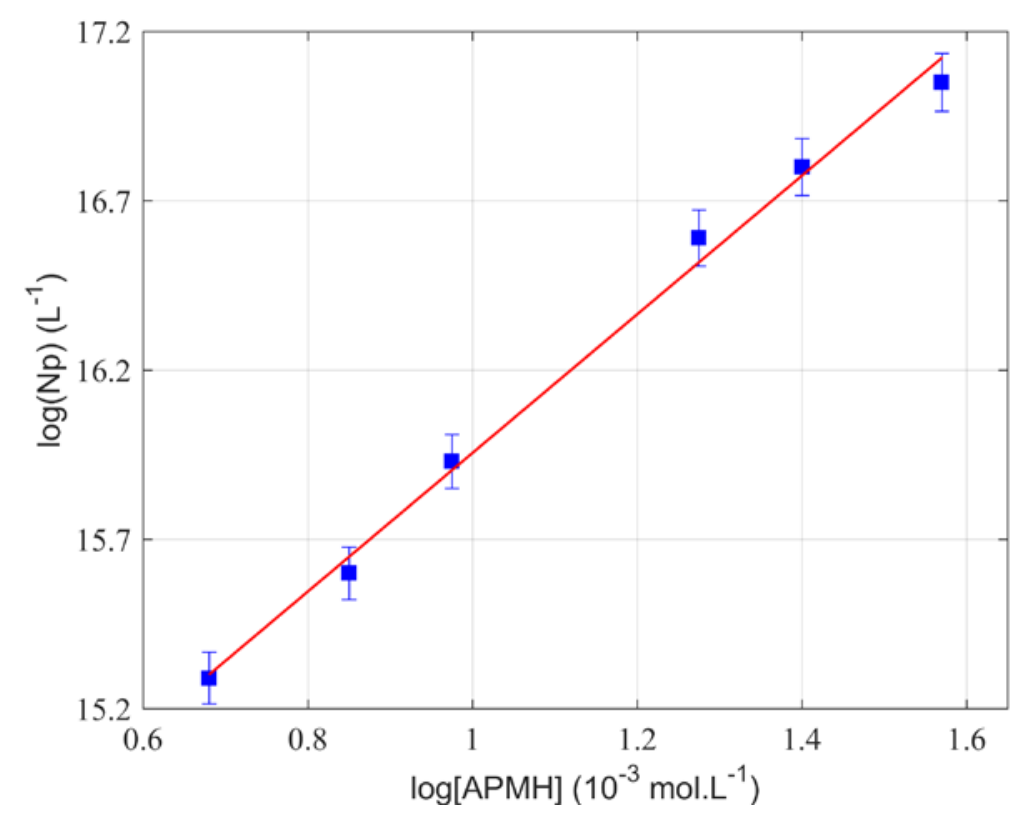

Figure 8: Total particle number variation versus ionic monomer concentration in log-log scale for a constant initiator concentration. 
The determined coefficient $\beta$ from the slope of $\log \left(\mathrm{N}_{\mathrm{p}}\right)$ versus $\log [\mathrm{A}]$ (illustrated in Figure 8) was found to be close to 2 . This value is slightly higher than the already reported values for other ionic monomers ( $\beta \sim 1.45$ for AEMH and VBAH $)^{33,34}$ reflecting the drastic effect of the charged monomer on particles formation during the nucleation step and their colloidal stability. In fact, the presence of charged comonomer enhances not only the number of polymerization loci but also the critical size of the precursors and the electrostatic stability of the immature polymer particles.

The final particle size, the particles number, the initial polymerization rate variation as a function of ionic charged comonomer concentration are in good agreement with the influence of addition of charged monomer on the coagulative nucleation polymerization mechanism of the classical free-emulsion polymerization in batch conditions as evidenced by few reported works. $^{18,33,34}$

In all reported studies, the water-soluble polymer formation has been generally neglected and the particles yield has been considered. The utilization of water-soluble comonomer in soapfree emulsion polymerization affects not only the polymerization process and the colloidal properties of the formed particles but also the amount of the water-soluble polymer (WSP). In fact, the increase in the ionic monomer concentration enhances the water-soluble polymer formation from 6 wt. \% to $20 \%$ as shown in Figure 9. The increase of the WSP amount versus APMH concentration can be principally attributed to the chemical composition and the solubility parameter of the ionic comonomer. In fact, the WSP chains should exhibit; (i) low molecular weight or (ii) rich APMH sequence. 


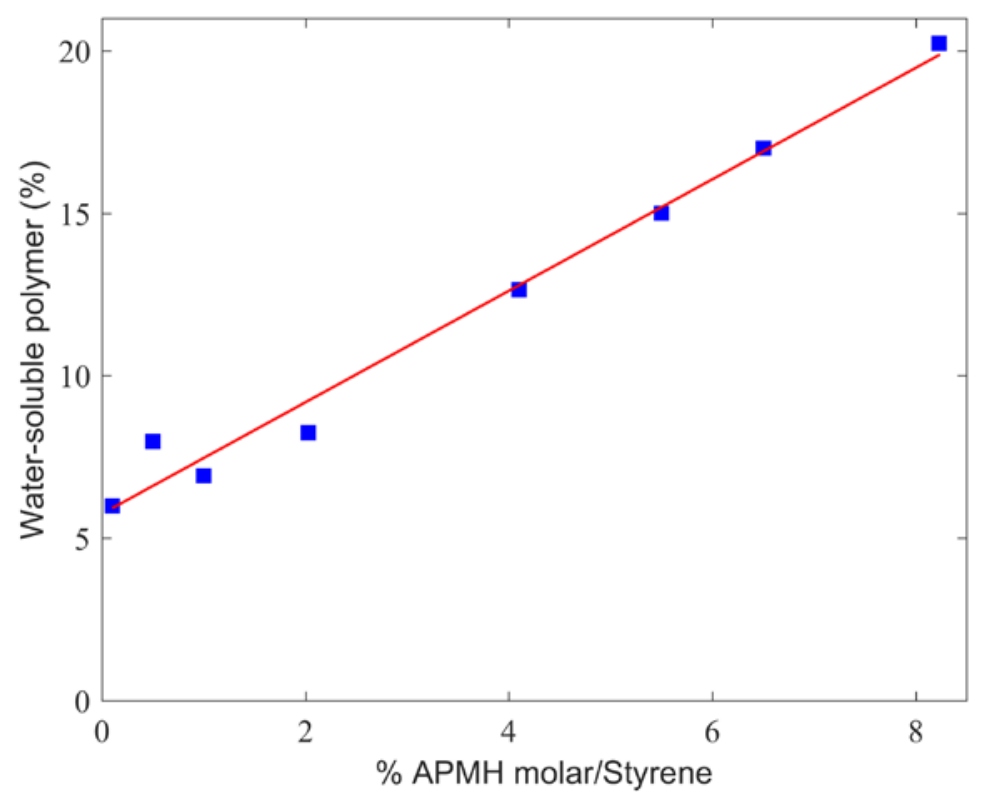

Figure 9: Influence of APMH on water-soluble polymer amount formation (wt.\% referred to the total solid content i.e. WSP and latex particles).

\subsection{Properties of Particles}

\subsubsection{Surface charge density evaluation}

The presence of functional groups on the particles surface, originated from the APMH monomer, is particularly interesting for the application of such particles in the biomedical field. Therefore, the total determination of all the reactive groups is needed to evaluate the incorporation yield of the ionic monomer. In this case of batch emulsion polymerization, only the surface functional groups are titrated.

The amounts of accessible surface amino groups from the functional monomer are directly determined using SPDP titration method as explained in the experimental part. The determined results are reported in Figure 10(a), in which the surface charge density (amidine and amine groups) is taken as function of the ionic monomer concentration. The surface 
charge density expressed in $\mu \mathrm{mol} . \mathrm{m}^{-2}$ was found to decrease with increasing the APMH concentration in the polymerization reaction medium. Such behavior can be explained by the drastic increase of the specific surface area (i.e. decrease in particle size) as illustrated in Figure 10(b). Whereas, the charge number per gram is found to increase with increasing the amount of APMH since the number of particles per unit of mass is increasing.
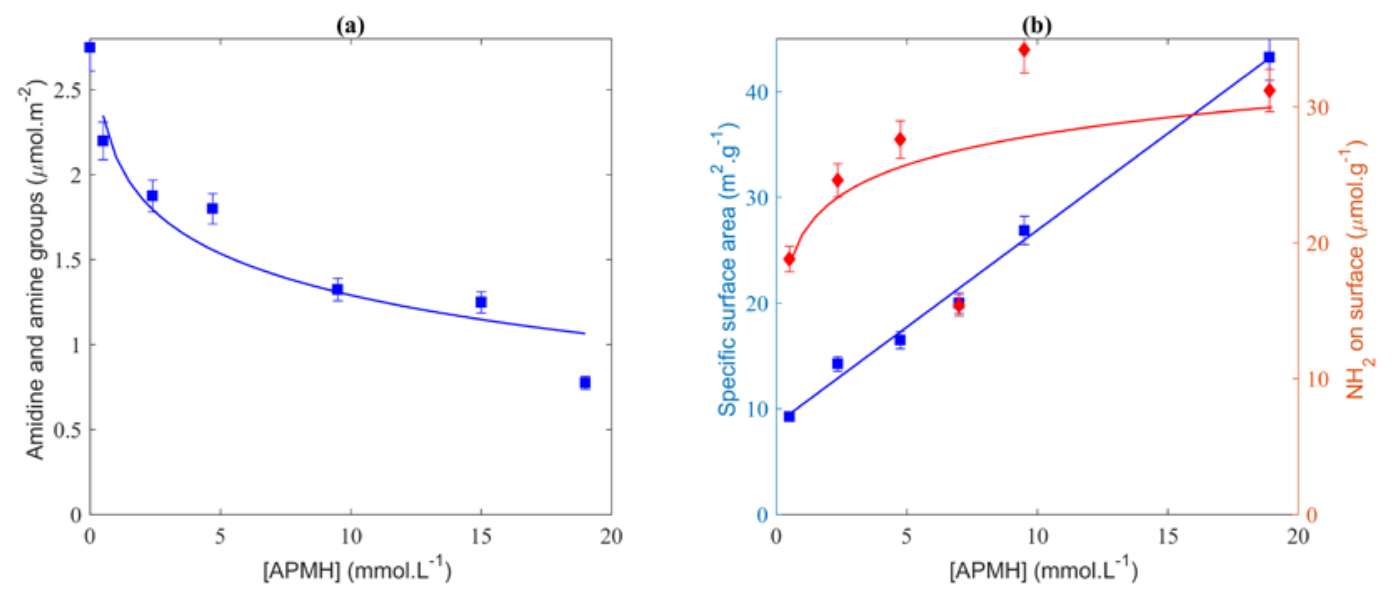

Figure 10: (a) Variation of the surface charge density $\left(\mu \mathrm{mol} \mathrm{m} \mathrm{m}^{-2}\right.$ ) and (b) variation of the specific surface area and $\mathrm{NH}_{2}$ density $\left(\mu \mathrm{mol} \mathrm{g} \mathrm{g}^{-1}\right.$ ) as a function of APMH concentration introduced in the batch polymerization recipe.

\subsubsection{Electrokinetic study}

The zeta potential of the prepared latexes was calculated from the measured electrophoretic mobility by using the Smolochowski's approximation. ${ }^{44}$ The deduced zeta potential exhibits a positive plateau below $\mathrm{pH}=8$, revealing the presence of cationic groups onto latex particle surface as expected and the results obtained are reported in Figure 11. The isoelectric point of the latex was found to be charge nature dependent. In fact, the isoelectric point shifts from $\mathrm{pH}$ $=8$ for amidine latex particles $(0 \% \mathrm{APMH})$ to $\mathrm{pH}=9.7$ for latex prepared using high amount of APMH (4\% molar ratio). This behavior can be attributed to an increase in the amine 
groups and a decrease in the amidine groups as already reported by Ganachaud et al. (1997). ${ }^{33,34}$ The isoelectric point of the latex prepared without APMH is close to the pKa 7.5 of the amidine groups (originated from the V50 initiator) and the latex prepared with high amounts of APMH monomer exhibits an isoelectric point close to the pKa 9.5 of the primary amine groups of the ionic monomer. In addition, the plateaus obtained at acidic $\mathrm{pH}$ are also found to be polymerization recipe-dependent. In fact, the maximum zeta potential increases with increasing the ionic monomer amount, which explains the efficiency incorporation of the functional monomer (APMH). In all cases, the observed zeta potential revealed the presence of carboxylic groups that might be originated from the hydrolysis of amidine groups at basic $\mathrm{pH} .{ }^{45}$

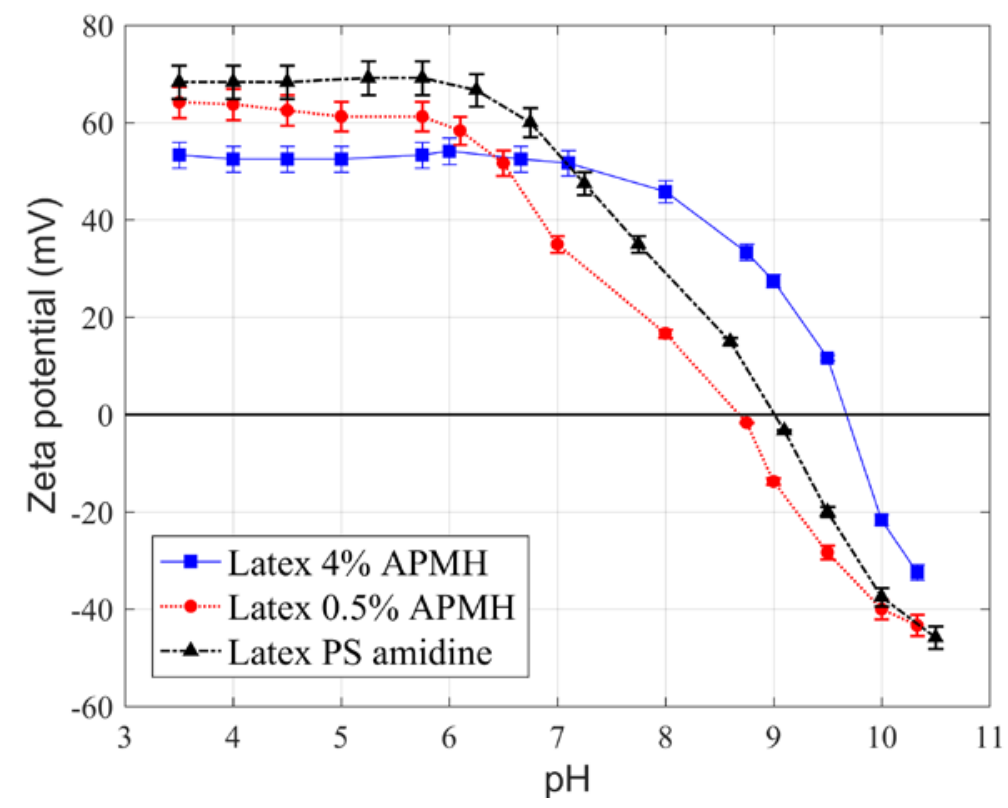

Figure 11: Zeta potential (mV) of three different latexes, with various APMH concentrations, as a function of $\mathrm{pH}$ in $10^{-3} \mathrm{M} \mathrm{NaCl}$ at $20^{\circ} \mathrm{C}$.

\section{CONCLUSION}


Soap-free emulsion polymerization of styrene with n-(3-aminopropyl) methacrylamide hydrochloride (APMH) cationic monomer offers a convenient mean to produce polystyrene latex particles bearing reactive amine groups. By controlling the polymerization conditions, and more particularly the concentration of $\mathrm{APMH}$, the size, the shape and the values of functionalization rate of the particles can be modulated in order to fit their biological needs (biological molecules immobilization). When the APMH concentration increased from $0.5 \%$ to $8.0 \%$ : i) Particle size decreased from $600 \mathrm{~nm}$ to $100 \mathrm{~nm}$ and ii) Initial polymerization rate $\left(\mathrm{R}_{\mathrm{p}}\right)$ and particles number $\left(\mathrm{N}_{\mathrm{p}}\right)$ increased in accordance with the Smith-Ewart theory for soapfree styrene emulsion polymerization with a hydrophilic functional comonomer. Finally, colloidal and electrokinetic studies were carried out to assess the surface charge density evolution with the concentration of APMH and to figure out stability domain of the formed nanoparticles with respect to the $\mathrm{pH}$ of the medium. Colloidal stability of the synthesized amine-functionalized polystyrene particles was enhanced upon introducing and increasing APMH monomer in the polymerization recipe in a wide range of $\mathrm{pH}$ (more particularly at acidic, neutral and highly basic media). This, in turn, makes these particles suitable for biomolecules immobilization.

\section{ACKNOWLEDGEMENT}

This work was carried out in the CNRS-BioMérieux laboratory and extended afterwards to the LAGEPP CNRS-University of Lyon1 Laboratory.

\section{REFERENCES}

1. Asua, J. M. In Polymer Reaction Engineering of Dispersed Systems: Volume II, W. Pauer, Ed.; Springer International Publishing, Cham 2018; pp. 1-22.

2. Çamli, T., Tuncel, M., Şenel, S., Tuncel, A. J. Appl. Polym. Sci., 2002, 84, 414-429. 
3. Unsal, E., Camli, S. T., Senel, S., Tuncel, A. J. Appl. Polym. Sci., 2004, 92, 607-618.

4. Yu, B., Tian, C., Cong, H., Xu, T. J. Mater. Sci., 2016, 51, 5240-5251.

5. Rahman, M., Heng, L. Y., Futra, D., Ling, T. L. Nanoscale Res. Lett., 2017, 12, 474.

6. Jamshaid, T., Tenório-Neto, E. T., Baraket, A., Lebaz, N., Elaissari, A., Sanchís, A., Salvador, J.-P., Marco, M.-P., Bausells, J., Errachid, A., Zine, N. Biosensors, 2020, 10, 43.

7. Galperin, A., Margel, S. J. Polym. Sci. Part Polym. Chem., 2006, 44, 3859-3868.

8. Galperin, A., Margel, D., Baniel, J., Dank, G., Biton, H., Margel, S. Biomaterials, 2007, 28, 4461-4468.

9. Illert, P., Wängler, B., Wängler, C., Zöllner, F., Uhrig, T., Litau, S., Pretze, M., Röder, T. J. Appl. Polym. Sci., 2019, 136, 47571.

10. Slomkowski, S., Basinska, T., Miksa, B. Polym. Adv. Technol., 2002, 13, 905-918.

11. Wang, T., Wang, L., Li, X., Hu, X., Han, Y., Luo, Y., Wang, Z., Li, Q., Aldalbahi, A., Wang, L., Song, S., Fan, C., Zhao, Y., Wang, M., Chen, N. ACS Appl. Mater. Interfaces, 2017, 9, 18619-18625.

12. Song, S., Zhang, W., Hu, Z., Zhang, Z. Colloids Surf. Physicochem. Eng. Asp., 2009, 348, 1-8.

13. Lee, C.-F., Young, T.-H., Huang, Y.-H., Chiu, W.-Y. Polymer, 2000, 41, 8565-8571.

14. Shegiwal, A., Wemyss, A. M., Schellekens, M. A. J., Bont, J. de, Town, J., Liarou, E., Patias, G., Atkins, C. J., Haddleton, D. M. J. Polym. Sci. Part Polym. Chem., 2019, 57, E1-E9.

15. Tamai, H., Hasegawa, M., Suzawa, T. J. Appl. Polym. Sci., 1989, 38, 403-412.

16. Nagai, K., Ohashi, T., Kaneko, R., Taniguchi, T. Colloids Surf. Physicochem. Eng. Asp., 1999, 153, 133-136.

17. Yamaguchi, K., Taniguchi, T., Kawaguchi, S., Nagai, K. Colloid Polym. Sci., 2002, 280, 942-948.

18. Ganachaud, F., Mouterde, G., Delair, T., Elaïssari, A., Pichot, C. Polym. Adv. Technol., 1995, 6, 480-488.

19. Cousin, P., Smith, P. J. Appl. Polym. Sci., 1994, 54, 1631-1641.

20. Miraballes-M artínez, I., Forcada, J. J. Polym. Sci. Part Polym. Chem., 2000, 38, 42304237.

21. Miraballes-Martínez, I., Martín-Molina, A., Galisteo-González, F., Forcada, J. J. Polym. Sci. Part Polym. Chem., 2001, 39, 2929-2936.

22. Musyanovych, A., Adler, H.-J. P. Langmuir, 2005, 21, 2209-2217.

23. Liu, X. Y., Zheng, S. W., Hong, R. Y., Wang, Y. Q., Feng, W. G. Colloids Surf. Physicochem. Eng. Asp., 2014, 443, 425-431.

24. Nestorson, A., Neoh, K. G., Kang, E. T., Järnström, L., Leufvén, A. Packag. Technol. Sci., 2008, 21, 193-205.

25. Pichot, C. Curr. Opin. Colloid Interface Sci., 2004, 9, 213-221.

26. Ramos, J., Forcada, J., Hidalgo-Alvarez, R. Chem. Rev., 2014, 114, 367-428.

27. Covolan, V. L., Mei, L. H. I., Rossi, C. L. Polym. Adv. Technol., 1997, 8, 44-50.

28. Forcada, J., Hidalgo-Alvarez, R. Forcada, J., Hidalgo-Alvarez, R. http://www.ingentaconnect.com/content/ben/coc/2005/00000009/00000011/art00004 (accessed March 23, 2020). 
29. Li, P., Zhu, J., Sunintaboon, P., Harris, F. W. J. Dispers. Sci. Technol., 2003, 24, 607613.

30. Molday, R. S., Dreyer, W. J., Rembaum, A., Yen, S. P. J. Cell Biol., 1975, 64, 75-88.

31. Quash, G., Roch, A.-M., Niveleau, A., Grange, J., Keolouangkhot, T., Huppert, J. J. Immunol. Methods, 1978, 22, 165-174.

32. Basinska, T., Slomkowski, S. Colloid Polym. Sci., 1995, 273, 431-438.

33. Ganachaud, F., Sauzedde, F., Elaïssari, A., Pichot, C. J. Appl. Polym. Sci., 1997, 65, 2315-2330.

34. Sauzedde, F., Ganachaud, F., Elaïssari, A., Pichot, C. J. Appl. Polym. Sci., 1997, 65, 2331-2342.

35. Delair, T., Marguet, V., Pichot, C., Mandrand, B. Colloid Polym. Sci., 1994, 272, 962970.

36. Charreyre, M.-T., Razafindrakoto, V., Veron, L., Delair, T., Pichot, C. Macromol. Chem. Phys., 1994, 195, 2153-2167.

37. Ramos, J., Martín-Molina, A., Sanz-Izquierdo, M. P., Rus, A., Borque, L., Hidalgo-Álvarez, R., Galisteo-González, F., Forcada, J. J. Polym. Sci. Part Polym. Chem., 2003, 41, 2404-2411.

38. Ramos, J., Forcada, J. J. Polym. Sci. Part Polym. Chem., 2005, 43, 3878-3886.

39. Musyanovych, A., Rossmanith, R., Tontsch, C., Landfester, K. Langmuir, 2007, 23, 5367-5376.

40. Grabs, I.-M., Schmidt-Naake, G. Macromol. Symp., 2009, 275-276, 133-141.

41. Fuchs, A.-K., Syrovets, T., Haas, K. A., Loos, C., Musyanovych, A., Mailänder, V., Landfester, K., Simmet, T. Biomaterials, 2016, 85, 78-87.

42. Chern, C. S. Prog. Polym. Sci., 2006, 31, 443-486.

43. Giannetti, E. Macromolecules, 1990, 23, 4748-4759.

44. Hunter, R. J. Zeta Potential in Colloid Science: Principles and Applications, Academic Press, 2013.

45. Ganachaud, F., Bouali, B., Véron, L., Lantéri, P., Elaïssari, A., Pichot, C. Colloids Surf. Physicochem. Eng. Asp., 1998, 137, 141-154. 